\title{
Recreating Herschel's Actinometry: An Essay in the Historiography of Experimental Practice
}

\section{Citation}

Voskuhl, Adelheid. 1997. Recreating Herschel's actinometry: An essay in the historiography of experimental practice. The British Journal for the History of Science 30(3): 337-355.

\section{Published Version}

doi:10.1017/S0007087497003129

\section{Permanent link}

http://nrs.harvard.edu/urn-3:HUL.InstRepos:3716614

\section{Terms of Use}

This article was downloaded from Harvard University's DASH repository, and is made available under the terms and conditions applicable to Other Posted Material, as set forth at http:// nrs.harvard.edu/urn-3:HUL.InstRepos:dash.current.terms-of-use\#LAA

\section{Share Your Story}

The Harvard community has made this article openly available.

Please share how this access benefits you. Submit a story.

\section{Accessibility}




\title{
Recreating Herschel's actinometry: An essay in the historiography of experimental practice
}

\author{
ADELHEID VOSKUHL*
}

... and signs and the signs of signs are used only when we are lacking things.

Brother William of Baskerville in The Name of the Rose by Umberto Eco

Recreating, as part of doing history, can be a way of reflecting what creating is as part of science. Discussions revolving around historical understanding of the scientific enterprise have recently included strong commitments to turn scientific practice into one of the main objectives of historical study. One specific methodological approach to face up to this assignment is integrating the reconstruction and reperformance of past experiments into the historical analysis of the doing-part in science. This paper deals with the doing-part in history, that is, with the historiographical consequences that might stem from this reconstruction and reperformance of past experimentation. In the course of a four-month period of research I worked with a replica of the so-called 'actinometer', an instrument to measure the intensity of solar radiation, which was invented by John Herschel in 1824. On the basis of this example, I try to trace how recent performances of experimental activities can contribute to historical understanding of human agency in scientific practice.

\section{SIGNS OF THINGS - THE HISTORIOGRAPHY}

A historical study that includes the reperformance of an experiment provides two different kinds of sources: 'practical', non-textual sources and theoretical, textual ones. Several historians have suggested reconstructing and reperforming historical experiments as an additional information resource for the history of past experimentation. David Gooding has used his students' replications of Michael Faraday's experimental work on

\footnotetext{
* Department of History and Philosophy of Science, University of Cambridge, now Department of Physics, University of Oldenburg, 26111 Oldenburg, Germany.

This study is the result of co-operation between the Department of History and Philosophy of Science, University of Cambridge, and the Research Group of Higher Education and History of Science at the Physics Department, University of Oldenburg. The modern replica of the actinometer was constructed at the University of Oldenburg's workshop. I am very grateful to Otto Sibum for inspiring the project and supervising its realization. I am particularly indebted to Falk Rieß, Christian Sichau and Hans Boettcher from Oldenburg and to Nick Jardine and Simon Schaffer from Cambridge. The libraries of the Harry Ransom Humanities Research Institute, University of Texas at Austin, and of the University of St Andrews, Scotland, permitted use of their resources. I thank Annemarie Maurer for taking the photographs of my experimental activities and for permission to reproduce them.
} 
electromagnetic forces, and Otto Sibum has presented a historical study of Joule's work on the mechanical equivalent of heat that is based on his own performance of this experiment. ${ }^{1}$

In what sense do these performances serve as sources for historical research? Both Gooding and Sibum are in search of non-verbal, non-articulate and preconceptual elements of experimental activity. They are both committed to dealing with the actual practice, and they are both struggling with incomplete sources and with the inherent confinements of representing action in texts. In using practical experimental work in their study, however, the two historians apply different ways of using a performance and representing their results. Gooding uses his students' replication of the sequences of Faraday's experimental work on 3 September 1821 - the day when Faraday succeeded in the experimental realization of the circular forces between magnetized needles and wires carrying electric currents. Gooding represents the understanding that these performances yield with the help of 'experimental maps': flow charts of the series of experimental occurrences, of the process character, of the actions, of the objects and their ontological status. ${ }^{2}$ These maps are meant to be a tool to convey the nature of experimental practice in its contingency and uncertainty, in its procedural character and its distinctive interplay of thought and action.

Sibum, in contrast, uses his own replicatory work on the mechanical equivalent of heat. ${ }^{3}$ From these performances, he develops the term 'gestural knowledge' to enunciate and conceptualize his performance as a historical source. ' 'Gestural knowledge' refers to the sophisticated skills and insights he had to develop during his experimental work in order to succeed in performing the experimental operation properly. These skills and insights include proper timing, proper interaction with the set-up, proper behaviour between instrument readings, finding a suitable space for the set-up and the like. As to using this result historically, Sibum recontextualizes his gestural knowledge in the subsequent historical analysis by pointing to a historical context where skills, such as the ones he has acquired, or spaces, such as the one he found to be suitable, could have become habitual or available, respectively, for Joule.

Examining Gooding's and Sibum's uses of their practical sources and their results suggests three levels on which a performance can become part of historical research. First there is what could be called a 'technical level' of gaining additional information from reperforming scientific investigative pathways: both Gooding and Sibum fill gaps in records, identify false drawings or sketches in publications and obtain a better

1 D. Gooding, 'History in the laboratory: can we really tell what went on?', in The Development of the Laboratory (ed. F. James), London, 1989, 63-89; and H. O. Sibum, 'Reworking the mechanical equivalent of heat: instruments of precision and gestures of accuracy in early Victorian England', Studies in the History and Philosophy of Science (1994), 26, 73-106. Further case studies and historiographical analyses include L. Belloni, 'The repetition of experiments and observations: its value in studying the history of medicine (and science)', Journal of the History of Medicine and Allied Sciences (1970), 25, 158-67; H. Kragh, An Introduction to the Historiography of Science, Cambridge, 1987, ch. 5; T. Settle, 'Galileo and early experimentation', in Springs of Scientific Creativity (ed. R. Aris, H. Davis and R. Stuewer), Minneapolis, 1983, 3-20.

2 A comprehensive introduction into the 'pictography' of Gooding's experimental maps, together with useful examples of its application is to be found in D. Gooding, 'Putting agency back into experiment', in Science as Practice and Culture (ed. A. Pickering), Chicago, 1992, 65-112.

3 From 1992 to 1994, Sibum, assisted by Peter Heering, worked with a replica of Joule's paddle-wheel set-up to measure the mechanical equivalent of heat.

4 Sibum, op. cit. (1), 76. 
understanding about the technical details of the experimental work, such as which scientific device must have been used and in which space the experiment was performed. The second level is the 'level of awareness', standing for the result of reperformances, which both authors call 'getting an idea of the procedural character', 'realizing how complex experimentation is', 'realizing how difficult operations such as observing or reading temperature are', and 'how difficult it is to determine when an experiment ends'. On the awareness level, the historian recovers parts of the non-articulate and non-textual dimension of experimental work, and comprehends the limited historical accessibility of experimental practice in the past. On a third level, finally, a performance offers a valuable structure of historical explanation for a 'successful' experiment. This structure can be found in Sibum's study. With the help of his concept 'gestural knowledge', he develops a historical explanation as to how an experimenter's manipulation of the material world results in a scientific matter of fact. Skills and mastery in measuring temperature and controlling heat flows, for example, were pervasive in the nineteenth-century brewing culture of which Joule was part. On the basis of performance and the gestural knowledge gained, Sibum describes Joule's scientific work as the amalgamation of two diverse types of cultural influences, the brewers' culture and the natural philosophers' culture. Moreover, Sibum explains the establishment of the value of the mechanical equivalent of heat as the successful outcome of Joule's credibility as a natural philosopher.

Further to these analyses of performance, Adrian Wilson has recently suggested the historiographical concept of 'hermeneutic stances' to categorize the ways in which historians apprehend, study, interprete and evaluate their sources. ${ }^{5}$ He distinguishes three different 'hermeneutic stances' a historian can hold while studying documents. In the first and the second hermeneutic stance, documents are regarded as 'authorities' or 'witnesses to the past', respectively. They share an ontological commitment to the documents and to the status of historical knowledge: both stances require that the documents yield a direct record of the past, or in other words that there is an identity between the historian's objectof-knowledge, on the one hand, and some body or item of evidence, on the other. The epistemological consequence of this assumption for the structure of historical knowledge is that doing history would be a procedure of extraction and compilation of information, rather than an activity of interpretation and inference.

In specifying the distinctive character of his third hermeneutic stance, Wilson outlines a historiography that thoroughly reappraises the relationships between the historian, the resources and the resulting piece of historical knowledge. In the third hermeneutic stance, a historical source is regarded as the product, or effect, of a procedure that lies in the past rather than a witness, an authority, or a window to former centuries: the existence of a record of the past is not linked to its function as a document for a researching historian, but only defined through the past period that gave rise to its being. The appropriate question to pose when studying an item of evidence, for example, would thus be: how did this document come into being? or: how can one infer from its existence to proceedings in the past and what kind of historical explanation does this document allow?

Linking the three levels of using a performance as a historical source to Wilson's concept

5 A. Wilson, 'Towards an integrated historiography', in Rethinking Social History (ed. A. Wilson), Manchester, 1993, 293-335. 
of 'hermeneutic stance' offers a helpful way of describing the relationship between historians' recent performances and their interpretation of historical experimentation. Assuming that a performance can in this specific sense count as a source, the main problem to solve would be to overcome the flaw of the first and second stance: not to confuse historical knowledge with the insights a performance yields, and to shift the historical object-of-research away from the document or the performance itself towards an interpretation of textual and non-textual sources that has its foundation in the analysis of a historical context where documents and practices come into being. It is with these aims in mind that I undertook a series of practical measurements with a replica actinometer. To explicate the sense in which my performance has contributed to my historical analysis of one specific experimental practice, I will try to assume a version of the third hermeneutic stance, by way of reading any text on 'action' as the remnant of action rather than an account of action, and in reading it I will try to direct the attention to the history that made a written representation of practice possible.

\section{THINGS - FIVE WEEKS IN THE SUN'S LABORATORY}

In 1824 John Herschel invented the actinometer as a device for measuring the intensity of solar radiation. It consists of a large cylindrical glass vessel that is soldered at one end to a thermometer tube. The thermometer tube is terminated at the upper end by a ball that is drawn out to a point. The point is broken off in order to allow the user to leave the end open or to seal it, at will, with a piece of wax. The other end of the cylinder is furnished with a movable bottom. With the help of a screw device, the volume and capacity of the cylinder can thus be altered. The scale attached to the thermometer tube is divided into arbitrary, greatly enlarged degrees. If the cylinder is filled with dark liquid, the actinometer, in principle, is a 'thermometer of great delicacy' (Figure 1). ${ }^{6}$

The disproportionately large volume of the thermometer bulb in relation to its tube and the variable capacity of the cylinder are essential properties of the instrument. The size of the cylinder and the small bore of the tube render the actinometer so delicate that even a very slight elevation of temperature suffices to carry the liquid up the whole column. ${ }^{7}$ The function of the movable bottom is to make a long tube redundant: through changing the position of the screw, the measuring capability of the instrument is restored if the liquid goes into the upper ball or drops back into the cylinder.

One core segment of the actinometer's peculiar measurement process consists of three steps. First, the instrument is exposed to the sun for a minute (or a different short period of time) and the alteration of temperature is recorded at the end of this minute. Then the actinometer is covered for a minute by a screen of tin plate or the like in order to expose it to the shadow. The temperature change after this shadow minute is read off and recorded. Thirdly, the screen is removed again, the actinometer exposed to the sun for a minute, and the increase read off and recorded (Figure 2).

\footnotetext{
6 J. Herschel, 'Instructions for making meteorological observations', in Report of the Committee of Physics and Meteorology of the Royal Society, London, 1840, 59.

$7 \mathrm{~J}$. Herschel, 'Explanation of the principle and construction of the actinometer', Report of the Third Meeting of the British Association for the Advancement of Science, London, 1834, 379.
} 
(a)

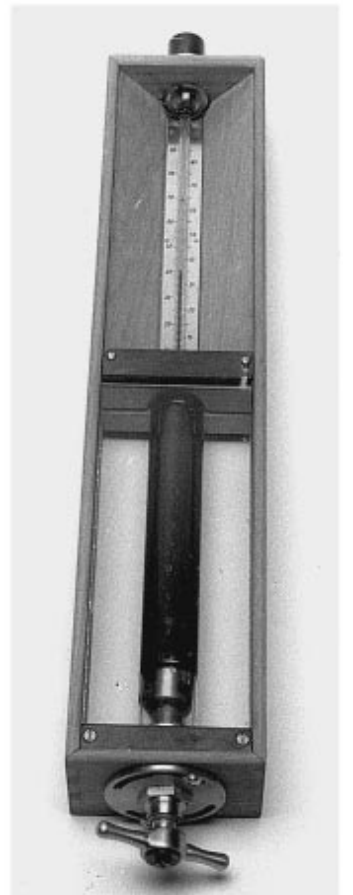

(b)

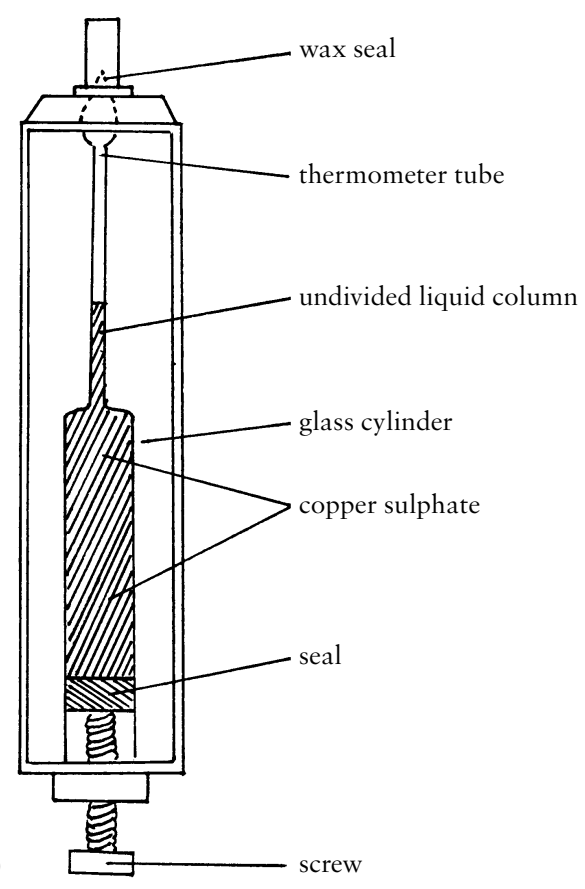

Figure 1. (a) The replica actinometer used for the study. Constructed by the University of Oldenburg's workshop. (b) The components of the actinometer.

\section{Phase 1. Encountering an object - confusion}

The first two working phases with the actinometer are indoor phases. I encounter and approach the object by touching it, taking it apart, putting it together again, experiencing what I can do with it, how I can manipulate it and change it. This involves performing operations that eventually become crucial routine within the measurement process, such as removing the screw device at the bottom of the instrument, filling the instrument with dark liquid, and reattaching the screw properly. This operation precedes every single measurement series.

Having filled the instrument with the measurement liquid, I face the first major technical problem. The seal, made of a leather collar, is not leak proof. It does not allow me to pump the air bubbles, which remain in the cylinder after I have filled the instrument, up into the glass ball. If I try to drive the air out of the cylinder by holding the instrument upright and turning the screw, the seal does not push the liquid upwards but it just passes 'through' the liquid, causing a big mess of dripping, corrosive, copper sulphate solution. ${ }^{8}$

8 According to the instructions, there must not be any air bubbles left in the cylinder. The air is supposed to be removed with the help of the routine described. Instructions for the actinometer's use are to be found in L. F. Kämtz, Lehrbuch der Meteorologie, 3 vols., Halle, 1836, iii, 15-20, J. Herschel, op. cit (6), 58-66, and A Manual of Scientific Inquiry; Prepared for the Use of Officers in Her Majesty's Navy; and Travellers in General, 2nd edn, London, 1851, 299-309. 


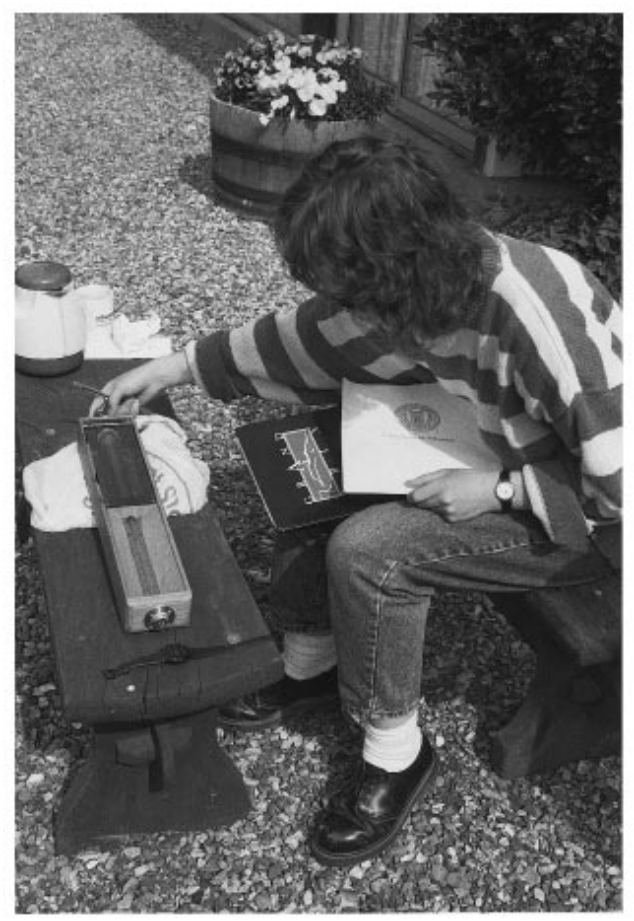

(a)

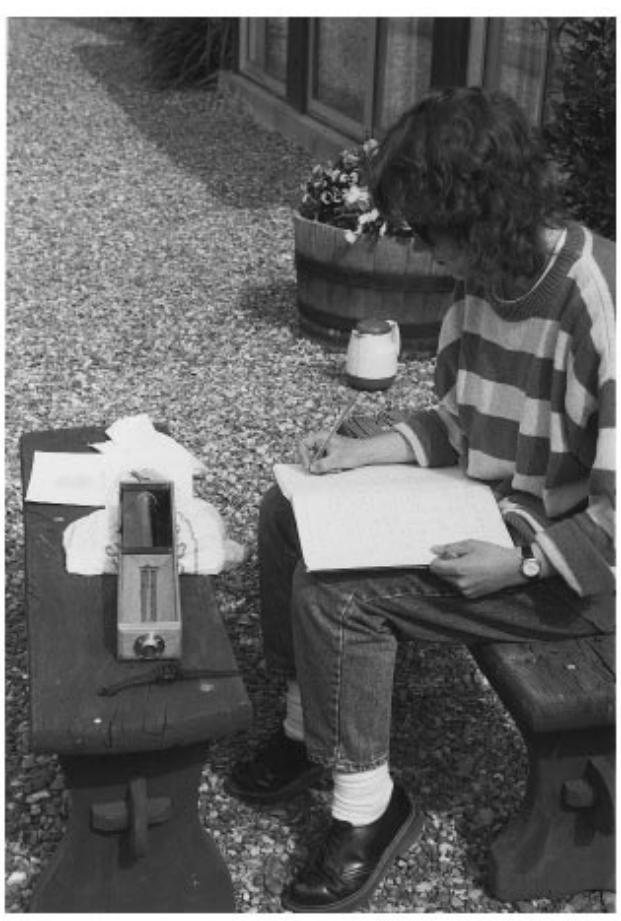

(b)

Figure 2. The author as part of the experimental process. (a) Readjusting the screw while measuring sunshine. (b) The activity of reconstructing practical data as a set of notes. Photographs courtesy of Annemarie Maurer.

I solve the seal problem by leaving a remnant of air in the cylinder and driving it up to the bottom part of the cylinder, next to the screw device. There, the air does not disturb the liquid's transition from the cylinder into the thermometer tube. Thus, I find a way of bypassing the instructions and work out a routine that enables me to avoid the messy affair of the seal. This routine allows me to cushion the first technical problem.

The main assignment of this preparatory phase is getting the instrument into a state of reasonable starting conditions for measurement: the top of an undivided liquid column in the thermometer tube has to be somewhere in the middle of the scale, the glass cylinder containing no air except right at the bottom next to the screw and preferably no liquid in the glass ball. This turns out to be a challenging task. It means learning how to fill the instrument properly (pouring in quickly enough, getting the dosage right, etc.) and how to create an undivided liquid column in the thermometer tube despite the complex interaction between air and liquid in the glass apparatus. Owing to the flawed seal, any manipulation of the screw causes a disturbance in the air-liquid balance. Once the instrument is filled, I find it impossible to manipulate the liquid in the apparatus in any determinable way.

The first phase of my work with the actinometer ends in serious confusion and frustration. The instrument exhibits a stubborn resistance to my efforts to handle and 
control it. I cannot see the slightest indication that, at some stage, I will have turned the artefact before my eyes into a measurement device for solar radiation.

\section{Phase 2. First major closure}

In the second phase of experimentation I devise strategies for avoiding or managing the problems identified in the first. These strategies result in controlled manipulation of the actinometer into a state suitable for starting measurement. What is 'controlled manipulation' and how does it come into being? I am trying to become familiar with the instrument in certain characteristic situations. What does the liquid in the cylinder and in the tube look like immediately after I filled it? How does this change with different 'strategies' of filling it (speed, position, etc.)? How do the air bubbles in both the cylinder and the tube behave within the first five minutes after filling it? How does this depend on the position of the instrument after filling it (horizontal, or the proper vertical position)?

After a sufficient number of trials I identify favourable conditions for preparing the actinometer for measuring: while filling, I hold it in a vertical position. I fill the instrument as quickly as possible, for the liquid then does not flow into the thermometer tube. Positioning the actinometer in a horizontal position makes all the air in the cylinder form one bubble, which places itself immediately under the surface of the cylinder, somewhere between the screw and the soldered joint at the tube. In this stable, horizontal position, I can make use of the screw without causing too much leakage.

The familiarity I acquire in handling the actinometer in this pre-experimentation phase stems from a peculiar learning process: vicissitudes of trial and error, of handling and observing, of fixing and repairing, of familiar and unfamiliar situations, of transforming unfamiliarity into familiarity, of confidence and frustration, of opening and closing situations, of shaping and moulding and altering my 'sense' of appropriate interaction with this object. ${ }^{9}$ As a result, 'controlled manipulation' consists of elaborating a reproducible, determined sequence of manipulations that gives rise to conditions of regular instrumental indication. This is an interesting paradox of the emergence of control and predictability from chaos and failure, which is going to recur each time I master a further ingredient of controlled measurement.

At the end of phase two I have acquired sufficient control over the instrument to prepare it for measurement procedures. This is the first major closure of my 'working system' with the actinometer. 'Openness' and 'closedness' are two essential properties Harry Collins attributes to systems of experimental work. Attempting to categorize different mechanisms that lead to closure of systems, he emphasizes the role of replicable phenomena in this process. ${ }^{10}$ This is exactly my way of coping with the open, chaotic system in phases one

9 By 'sense' I mean a quality of a practical process of mastering, similar to the ones Bourdieu describes. He considers the transition of practical mastery in practice to be a process that contains further components to trial and error, even if they are not passed on through discourse or consciousness. See P. Bourdieu, Outline of a Theory of Practice, Cambridge, 1977, 87-8. The non-verbal and even unconscious elements of my mastery are by no means subordinated to those that seem more 'conscious' and cognitive.

10 H. Collins, Changing Order. Replication and Induction in Scientific Practice, London, 1985, ch. 2. 
and two: I transform the disordered conditions in which I find myself into a predictable sequence of replicable events.

\section{Phase 3. Good day, sunshine - outdoors}

The transition to the open-air laboratory in the sun in the third phase requires further technical improvization to compensate for the weak points of the instrument's construction, in particular the seal and the closedness of the air-liquid system. I experiment with the seal by testing several kinds and thicknesses of leather, different numbers of leather collars and varying amounts of grease applied to both the leather and the cylinder in order to obtain a seal that is as tight as possible.

The result of these efforts is counterproductive: the cylinder breaks at one point when I insert a seal that is too big. Luckily, it is a relatively plain breakage: one small piece of glass falls out of the cylinder, and I can fix it sufficiently with a bandage of sellotape. Now, however, I have to balance carefully the delicacy of the broken glass on the one hand and the tightness of the seal on the other.

Having repaired the actinometer so that it seems usable and more or less properly functioning, I proceed to the first measurement series. As a result of working phase 2, I am at a point where I can manipulate the actinometer into the state of an undivided liquid column and controlled air bubbles in the cylinder. Then, I face the next obstacle. I completely fail in levelling the top of the liquid column at a point of my choice on the scale: any time I adjust it to, say, 50 degrees, it drops back into the cylinder within half a minute. Several trials of pumping the liquid back into the tube all fail in the same way: I do not succeed in keeping liquid up in the tube.

My first attempt to deal with this problem is based on the assumption that the irregular behaviour is connected with temperature adjustments of the actinometer in the sun. I therefore make sure from now on that whenever I experiment outside I leave it exposed to sunshine for half an hour after having filled it, to rule out irregularities due to temperature differences between the instrument and the surrounding air. Yet this strategy does not yield controlled conditions. Several trials and long period of exposure do not give rise to regular behaviour of the liquid in the glass apparatus.

My second attempt to regain the closure of the system is to change the arrangement of the instrument into an inclined position, the bottom slightly higher than the top. This drives the remaining air bubble right to the bottom of the cylinder, next to the screw, and allows the liquid to flow into the stem more easily. Despite this more advantageous position, there is still no liquid to be found in the thermometer tube after a period of fifteen minutes.

The instrument's behaviour, however, matches the enigmatic and absurd impression I have gained so far: I have not got the slightest indication that the actinometer is a measurement device for solar radiation, for it does not at all exhibit reactions that would suggest this function. In the course of exposing the instrument to the sun for periods up to several hours, nothing significant happens. The only recurring, predictable event is that the liquid drops back into the cylinder after a certain amount of time, despite the 
actinometer being permanently bathed in sunshine. In this period of experimentation, I find the actinometer in a state where it is not visibly affected either by the sun or by me.

As a third attempt to make the instrument react to my interventions, I go back to using the screw, devising the following experiment: I perform trials to pump the liquid up into the tube, to three different values: 20, 40,60 degrees. At any time, it takes the liquid about half a minute to drop back into the cylinder. I try to get it up to 100 degrees: Now it only needs six seconds to disappear again. I try a second time to go up to 100 degrees, yet the capacity of the screw is used up just before I reach 70 degrees, and still there is no liquid in the tube. While completely unwinding the screw again (to regain the full capacity), suddenly, the liquid appears in the tube. It stabilizes at 44 degrees.

During these 'pumping experiments', eventually it occurs to me that the remnant of air in the glass apparatus could be the source of the trouble. The air interacts with the liquid in ways beyond my control, pressing the liquid back into the cylinder, generating bubbles in the stem, and weakening pressure in the cylinder. On the basis of this observation, I examine to what extent the actinometer's behaviour is dependent upon the sealing of the glass apparatus. I devise and perform experiments with and without a wax seal at the top of the glass ball. Although the printed sources explicitly say that the actinometer is ready for measurement once the glass cylinder is filled and the point sealed with wax, I aim at finding out what difference that makes and how far I can rely on such instructions. ${ }^{11}$ For despite explicitly contrary instructions, I cannot see any other way of getting the actinometer into a state that allows me to interact with it.

The actinometer stands at 27 degrees when I start my measurement series. I attach the sealing wax. With the screw I pump up the liquid to 50 degrees. After one minute, the column has fallen back into the cylinder. I pump it up to 100 degrees: it slowly drops back into the cylinder. Now I take off the wax, the actinometer stays at 31 degrees. I pump the liquid up the tube: the liquid reacts much more quickly to turning the screw. It takes one revolution to get the liquid up to the top. In fact, it does not feel like 'pumping' the liquid any more. I can easily control the liquid now and fix it at any position with a deviation of 1 degree at most. I try the values 90,50 and 10 degrees, and it easily stays at the point where I put it. In order to appraise what the influencing factors are that enable me to control the liquid I attach the wax again. And behold, the problems with fixing the liquid in a determined position reappear.

The first outdoors phase of desperate attempts to turn an object into a measurement tool results in a decisive skill: having made myself familiar with the actinometer's reactions to sunlight, with the intricacies of its technical peculiarities, and having developed appropriate operations and manipulations to respond to these properties, I have gained control over the instrument to an extent that allows performances of 'proper' measurements: I have gained a considerable degree of routine in the preparation process of an experimental session, I am capable of smoothly performing the sequence of putting the instrument's component parts together, arranging the other ingredients of the experimental setting (notebook, watch, tissues, screen), and pouring the measuring liquid into the actinometer so that there

11 The instructions I rely on are Kämtz, op. cit. (8), 15; Herschel, op. cit. (6), 59 and op. cit. (8), 301, and parts from the collection of Herschel's papers in the Harry Ransom Research Institute, University of Texas at Austin (hereafter Herschel Collection), nos. W 0078 and W 0079. 
is the least hassle with air bubbles. I can now base my first attempts at measuring on a properly arranged open-air lab. Moreover, I have made this prearrangement habitual: the sequence of operations is fixed, determined and replicable.

\section{Phase 4. Numbers - first proper measurement}

The very first measurement procedures I perform are simplified; they serve as a preliminary test of the instrument's reaction to being shaded. For after so much experience with 'irregular' behaviour, I constantly anticipate that something completely surprising will happen. I expect reopening of the system and breakdowns of all kinds, the collapse of my laboriously gained familiarity. Therefore I want to make myself step-by-step familiar with the measurement procedure in order to react properly to any complication and to trace the growing degree of complexity in my measurement habits.

So I expose the actinometer to the sun, level it at 45 degrees, simply shade it with my body, standing between the sun and the instrument, and observe without recording data. By means of this observation mode I perform several measurement series lasting five minutes on average, exposing and shading the actinometer in turn. The instrument does not show strong reactions: it falls and rises, respectively, by about 3 degrees. Nevertheless, I count this as a first success, observing indications that seem regular and reasonable to me. I close the system at a stage where I decide that my performing the core of actinometric measuring, the shading and exposing, does not lead to significant muddle.

After these first hints of a 'normal' actinometric measurement, I aim at consolidating this development by employing ways of shading the instrument with cardboard, recording the data in tables, and lengthening and stabilizing measurement series. Thus I perform my second mode of measurement series. The first one lasts four minutes, then I interrupt. The liquid shows irregular behaviour: it drops or rises by 7 degrees within half a second, then stays at the same position for half a minute, and the like. My idea about controlled experimentation with a measurement device would not allow indications of this kind to be proper measurement. I fail in producing data that I consider to be appropriate, and I ascribe this failure to my lack of skill in handling the apparatus properly. As a consequence, I deliberately open the system again. Reacting to irregularity, I intend to delete it, evolve more sophisticated skills, and thus produce more plausible indications.

The experience of the following measurements, however, makes me revise my understanding of this (causal?) relationship between 'regular data' and 'proper performance'. ${ }^{12}$ For despite numerous and diligent efforts to bestow a touch of regularity upon the instrument's indications, I cannot improve the 'quality' of the data. I decide to dismiss a causal connection between the quality of performance and the quality of data, and I now concentrate on producing measurement series and lengthening them, regardless of the resulting data. My attitude towards the relationship between myself and the regularity of the instrument's indications undergoes a change during this phase. This results in a revision of my strategies of opening and closing my experimental system.

12 This causality is the basis of what Collins, op. cit. (10), terms the 'experimenter's regress'. His analysis of experimental activity in scientific laboratories reveals that the quality of an experiment and its correct conduct is circularly defined through the correct outcome. 
Irregular behaviour and counter-intuitive data, normally, would seem the exemplary incidents that give rise to reopening a system and eliminate potential causes of the irregularity. My failure in finding such a cause and also my previous experiences of the underlying chaos in experimentation force me into loosening the bonds of the experimenter's regress.

Performing lengthy measurement series and registering their results in proper recording tables requires a lot of practice. I realize how quickly one minute elapses. Within this minute many things have to happen, have to be performed, such as watching the clock, shading the instrument, observing the sun, observing the liquid, taking notes of weather conditions and other peculiarities, and recording data. I have to acquire skills to keep my numerous 'lab-ingredients' under control for long periods of time when a measurement series can last over an hour.

Building up the aptitude of these lengthy performances results in an underlying paradox of acquiring control over experimental procedures. My main strategies of coping with complex and demanding experimental procedures have included becoming familiar with the instrument's behaviour, developing routines of controlling this behaviour, gradually simplifying my working habits, and focusing my attention on elements that turned out be crucial for controlling the set-up. A particularly interesting example for my success is the preparation of recording tables before a measurement session. It is, however, only half of their function that they enable me to order my performance and to lengthen my measurement series. For simultaneously they suppress my concentration on the various incidents in my sun-lab and make me confine my attention to a single aspect of experimentation: to recording data, to extracting nothing but a number. In doing so, however, I lose sight of all the other elements that I have come across so far and that also constitute experimental practice. The interface between a human and an instrument is made of more activities than recording tables can represent. My efforts in the performance of actinometric measurements have led to uncovering precisely what else can be said about an experimenter's work other than observing and quantifying natural phenomena.

\section{Phase 5. Meaning}

The next breakdown of my open-air lab emerges from the last closure: now that I have managed to build up habits and skills for performing lengthy measurements and can stay out in the sun's lab for several hours, there are changes in the actinometer's behaviour. When the actinometer has been exposed to the sun for a period of several hours, at some point it will suddenly react much more strongly to sun and shade. So far, when the measurement series covered only two hours or so, the changes normally never exceeded 25 degrees. Now, the indication falls or rises by more than 80 degrees during one minute.

The measurement sessions of the following days confirm this finding. I choose varying weather conditions to identify a tendency in the instrument's indications. In bright sunshine, around midday, the switch in the actinometer's reactions happens even earlier, for example the first series of one day shows changes of 3-10 degrees during one minute, while the second series, performed thirteen minutes later, uses the whole scale during one minute. On a day of relatively stable weather conditions over the course of eight hours, I 
perform measurements in the early morning and in the early afternoon. In the morning, the indications of shadow and sun minutes lie between 1 and 11 degrees. In the afternoon, however, the instrument's indications cover up to 70 degrees per minute.

During periods of slow reaction, I can perform measurement series of considerable length and I feel 'on top of things' for the first time in my experimentation work. I know where to direct my attention, I have enough time to observe, I have enough routine to keep everything else 'invisible', and I even start feeling bored, realizing how much tedium a routine measurement procedure involves. Only in this state do I succeed in restricting my concentration to the production of data. However, once the actinometer is exposed to the sun for a long time, or the measurements are being taken around midday, the whole business becomes really stressful.

The discrepancy between my morning and afternoon performances is a beautiful reflection of the difference between mastered performance and laborious experimentation. With the actinometer's sudden switch to stronger and quicker reactions, I am roused out of a calm rhythm of observing and recording, thrown into a new, open situation that requires a completely different mode of practical work: I have trouble reacting quickly enough to the actinometer's indications, I have trouble recording the data quickly enough, and, worst of all, I have to make use of the screw to regain the instrument's measuring capacity after the liquid has dropped back into the cylinder. Since the actinometer tends to drop more quickly during shadow minutes than it rises during sun minutes, I eventually end up performing three-step measurement series (one and a half minutes), then I have to readjust the actinometer.

My working phase ends with a success in performance and a failure in numbers: irregularity and unpredictability of data. Equal conditions do not necessarily bring about equal measurement values. In different measurement series, the actinometer's indications exhibit different degrees of 'inertia', and I fail in identifying a cause for this irregularity. Numerous measurement series in different conditions, different times of the day and different sky conditions do not result in my understanding of what causes the varying degrees of the liquid's inertia, let alone my aptitude to eliminate this element of irregularity and unpredictability. So far, any breakdown or failure or reopening of the system has resulted from the intrusion of a further, hitherto invisible and unknown, bit of the real world that demands my attention. Most of my attention, and my reactions to an unfamiliar phenomenon have led to a control or an elimination of this phenomenon. Absurdly, I fail in the last step, the most important one: I do not succeed in developing strategies to manipulate the conditions and the material in a way that yields what constitutes and defines a proper measurement performance: producing regular, meaningful numbers.

\section{SIGNS OF SIGNS OF THINGS - THE INTERPRETATION}

My practical work with the actinometer serves as an interpretive tool for the reading of textual representation of actinometric measurement. I encountered success and failure, chaos and order, gaining and loss of understanding, and I experienced how much labour and activity needs to be invested to establish measurement. This experience suggests a reading of the written material according to Wilson's third hermeneutic stance: a textual 
representation of experimental practice is the 'effect' and the product of human agency and human activity. ${ }^{13}$ This reading could bear two different meanings as to an interpretation of the text. First of all, an interpretation of a textual representation of experimental work on the basis of a performance directs the historian's attention strongly towards the practice and the experimenter as the 'causes' of the text's existence. Thus the practice and the processes of the text's emergence reappear in the reading. A text, read as the upshot of human activity, then, cannot be taken by itself to be an image or an accurate account or rendering of this activity. Instead, the reading requires to go beyond the literary account of practice, towards its origins, to the process of its development and to the past agent. Secondly, my activity and my agency in the experimental work turned out to be the effort of establishing order. In consequence, a reading of the product of that kind of activity (that is, the text) would happen with ontological commitment to agency that brings about order rather than with commitment to underlying order in experimental inquiry or underlying order in nature. ${ }^{14}$

Two decisive, textual remnants of the study of practical actinometric measurement from the nineteenth century are Herschel's published instructions for the use of the actinometer and his personal actinometer notebooks. Herschel communicated public instructions for the actinometer's use through the Royal Society's Report of the Committee of Physics and Meteorology, Relative to the Observations to be made in the Antarctic Expedition and in the Magnetic Observatories in 1840, and the Admiralty's Manual of Scientific Inquiry; Prepared for the Use of Officers in Her Majesty's Navy; and Travellers in General in 1851. His notebooks were unpublished. ${ }^{15}$ How should these sources be read? There is a considerable contrast between these two ways of representing actinometric measurement: in his private notebook, Herschel could record any instant of his work with the actinometer, regardless of an audience that had to be provided with meaningful and linear accounts. The notebooks bear protected records of an individual's labour on the establishment of a measurement procedure. Published instructions, on the other hand, demand linearity in a succession of actions and they demand a potential of replicability. They are published, multiplied, 'black-boxed' directions for the depersonified lay user.

It is particularly suggestive to cast the difference of public and private sources in terms of their degree of 'depth' or 'closeness' of what 'really' goes on in experimental practice. As Martin Rudwick has pointed out, however, the dichotomy between 'private' and 'public' textual resources is misleading in its implication of 'appropriate', 'close', 'deep' representations of an individual's practical inquiry on the one hand, and 'cleansed', 'shallow', 'superficial' representations on the other. ${ }^{16}$ With respect to the representation of action in writing, the deception might even be more serious by confusing the scale of

13 Wilson, op. cit. (5), 304.

14 Harry Collins similarly shifts the explanatory burden for ordered experimentation away from the regularity of the world towards the regularity of our institutionalized beliefs. See Collins, op. cit. (10), 147-8. For an elaboration of the term 'human agency' as an explanatory item of scientific change, see D. Gooding, Experiment and the Making of Meaning: Human Agency in Scientific Observation and Experiment, Boston, MA, 1990.

15 The Royal Society's Report and the Manual of Scientific Inquiry are referred to above in Herschel, op. cit. (6) and (8), respectively. The actinometer notebooks are part of the Herschel Collection, nos. W $0064-\mathrm{W} 0068$.

16 M. Rudwick, 'Charles Darwin in London: the integration of private and public science', Isis (1982) 73, 186-206, on 198. 
privacy with the scale from 'close' to 'rough' representation, and with the scale from 'thought' to 'action'.

The two underlying issues of the present discussion, the historian-source relationship and the thought-action dichotomy, mesh exactly at the point when it comes to epistemological problems in historical research on non-articulate action. Just because practical activity is difficult to access historically, it seems seductive to resort to a type of written text that is, at least, more 'private' and therefore 'closer' to the actual proceedings. One consequence of Wilson's third hermeneutic stance, however, is that such dichotomies and the supposed status of 'epistemologically privileged' information sources disappear. Wilson's suggestion implies that the bond between a document and historical knowledge becomes much looser. Neither past agents' thoughts nor their actions are immediately accessible by extracting the content of historical documents or by reperforming historical experiments.

\section{Herschel's actinometric notebooks}

Herschel mainly recorded the results of his actinometry in five notebooks. The earliest entries and numbers date from 1826. They are listed in notebook no. 5 where Herschel retrospectively wrote down an account of the incidents that stimulated him to devise an instrument for the measurement of solar radiation. This notebook, however, was written last, and it appears to have served as Herschel's personal chronicle of the first three years' work with the actinometer, from its invention in 1824 to July 1827. In notebook no. 1, then, the data continues three days after the last entry of notebook no. 5. The retrospective character is lost here: it seems to be a lab-notebook for real-time registration of experimental practice. It covers Herschel's work from 1827 to 1829. Notebook no. 2 does not serve exclusively as a record for actinometric measurement, but also serves for further meteorological study and register. It contains data from 1833 and 1834, the first two years of Herschel's five-year stay at the Cape of Good Hope. At the Cape of Good Hope, it was four times a year, at the equinoxes and the solstices, that Herschel made hourly meteorological observations, which included actinometric measurements. Notebook no. 3 continues with data from 1836, and it also shows records of Herschel's trials to calibrate the actinometer, just after the summer solstice of December $1836 .{ }^{17}$ Notebook no. 4 , from 1837, contains the final parts of his actinometric work at the Cape. After his return to England, however, Herschel realized that the actinometric data he had collected were useless, as he had not taken into account that the reduction of the data required a correction factor depending on the liquid's temperature. ${ }^{18}$

17 To calibrate the actinometer's indication, Herschel compared actinometer readings to changes in the temperature of water, when both were alternately exposed to the sun and shaded. Furthermore, he defined a unit for the measure of solar radiation, the 'actine', as 'the intensity of solar radiation which would melt a layer of ice 0.0000001 of a meter thick every minute'. For a comprehensive account of Herschel's efforts to determine the solar constant, see P. A. Kidwell, 'Prelude to solar energy: Pouillet, Herschel, Forbes and the solar constant', Annals of Science (1981), 38, 457-76. Herschel's records of the calibration are to be found in his 'Actinometer Notebook no. 3', Herschel Collection, no. W 0066.

18 Herschel mentions this omission somewhat laconically in the appendix of J. Herschel, Results of Astronomical Observations Made During the Years 1834, 5, 6, 7, 8, at the Cape of Good Hope, London, 1847. 
The recording tables and the additional entries in the notebooks are the main written representatives of Herschel's experimental action. Their analysis and interpretation against the background of my performance sheds light on various aspects of scientific practice. There are, first of all, two different renderings of experimental action. In notebook no. 5, the data are neatly arranged in recording tables and at regular intervals there are evaluations of the data in the form of synoptical tables and lists, together with some mathematical calculation. Also, Herschel makes detailed comments on the weather conditions, the brightness of the sun, strength of the wind, etc. Notebook no. 1, however, offers a different picture. Many entries are scribbled in pencil rather than written in ink, measurement tables and calculations are crossed out here and there, the tables fill a whole page, they are not prearranged, and ink- and pencil-written entries are written on top of each other. ${ }^{19}$ One page is no longer the record of a single 'inscription' process. ${ }^{20}$ The paper bears several time layers and the switches between recording data and their mathematical evaluation are not as regular as they used to be.

The comparison of organized, detailed recording with disorder and non-linear temporal succession not only points to the difference between real-time registration of action and retrospective rendition of action, but also mirrors two different qualities scientific practice can possess: scientific activity can be a linear, sequential, determinate, meaningful series of operations; yet scientific activity also figures as disordered, non-successive action, and it can lack meaning. ${ }^{21} \mathrm{My}$ practical understanding of actinometric measurement, gained from the performance, leads me to regard experimental practice as an activity that possesses both these qualities, and it leads me to weigh both the ordered and the chaotic dimension of scientific practice equally in my ontological judgement of what practice is. Encountering and analysing different sorts of 'inscribing practices', I apprehend them as accounts of both the goal-directed and the tinkering elements of activity, and as an account of the processes that transform chaotic action into meaningful data.

A second aspect of scientific practice and its written representation is suggested by the paradoxical nature of recording tables. A recording table is, on the one hand, the result of a successful transformation of chaos into order, and, on the other hand, the very entity that subsequently conceals the labour of this transformation and the labour that gave rise to its being. It makes invisible what originally led to its existence. ${ }^{22}$ How does my practical understanding contribute to the interpretation of recording tables? In my performance, I experienced prearranged recording tables to be indispensable, yet 'self-contradictory'

19 The look of this part of the measurement record, in fact, resembles the look of Herschel's travel journals. The function of Herschel's travel journals is, in contrast to many of his scientific notes or records of data, very close to what could be called 'real-time records' of experience or inquiry. The travel journals are part of the Herschel Collection, nos. W 0055-W 0063.

20 Bruno Latour develops the term 'inscription', in connection with 'mobility' and 'immutability', to account for the relevance of recording and registering in scientific practice, and to describe the role of mobile, stable representations on paper. See B. Latour, 'Visualization and cognition. Thinking with hands and eyes', Knowledge and Society (1986), 6, 1-40.

21 D. Gooding argues that all accounts of experimentation entail reconstruction and ordering, and that it is this reconstructive nature of thought and action that makes the 'actual' scientific practice inaccessible for historians. See Gooding, op. cit. (2), 76 and op. cit. (1), 71.

22 For the parallel paradox of invisible work and invisible equipment, see D. Gooding et al. (eds.), The Uses of Experiment, Cambridge, 1989, ch. 1. 
tools: they enabled me to create order in the setting of experimental inquiry, and they prevented me attending to the numerous other occurrences in my lab. The order required strong concentration on the 'right' things, on isolated aspects of my work. Isolation, however, goes together with loss of connections to the operations that render the open-air lab a closed stable system. The resulting simultaneous necessity and invisibility of agency now appears to be an inherent property of meaningful experimental inquiry.

The third aspect of scientific practice is about the ontology of 'normal measurement' and its reflection in textual rendering. Recording tables, in particular, suggest a state of stability and normality in the setting of experimental activity. The extemporary work I was performing in my experimental work, however, reflects a very specific idea of what normality in experimental work is. Its main part consisted of reacting to recurring breakdowns, of balancing, compensating, parrying, cushioning, of absorbing accidents, of rigging up and concocting things. The goal of these efforts was setting up conditions that allow me to perform 'normal' measurement procedures. The character, the existence, and the attainability of 'normal' conditions, consequently, is called into question: rather than normal conditions, they seem to be highly specialized and available only through diligent and patient experimental selection labour. The stability of inscriptions, however, conceals the temporality and the contingency of a state of order. Systems of experimental practice bear a potential for reopening as regularity and order can evade the system at any time. ${ }^{23}$ The inscription of a state of order, however, will remain in a state of order and is, in this sense, mute about the limits of regular and meaningful experimental action.

The last, and maybe most intricate, aspect of experimental practice in relation to private notebooks to be considered here is the issue of success. The actinometer was part of a scientific culture in the nineteenth century that prescribed the collection of huge amounts of data and their mathematical ordering and deduction. ${ }^{24}$ Bruno Latour argues that the purpose of this kind of activity is the plausible transformation of extensive natural phenomena into tangible, two-dimensional representations on paper. ${ }^{25}$ Furthermore, the scientific method generates a presupposition that makes this plausible transformation inevitable: diverse natural phenomena on various scales and diverse data gained from travelling long distances are linked together by the assumption that they are different effects of the same underlying principle. In the case of 'heat radiation', for example, Lambert, one of the first natural philosophers to study this phenomenon, extended his photometry based

23 The way in which Herschel himself tried to deal with the elusiveness of normality is beautifully illustrated in the introduction to the chapter on meteorology in the Manual of Scientific Inquiry, op. cit. (8). Here, he suggests how to set up favourable conditions for measurement and gives detailed instructions of how to accomplish regular registration of meteorological data on a sea voyage.

24 Detailed analyses of the features of nineteenth-century scientific culture include S. Brush, The Temperature of History, New York, 1978, 45-60; D. Miller, 'The revival of the physical sciences, 1815-1840', Osiris (1986), 2, 107-34; P. Lawrence, 'Heaven and earth - the relation of the nebular hypothesis to geology', in Cosmology, History, and Theology (ed. W. Yourgrau and A. Breck), New York, 1977; J. Cawood, 'The magnetic crusade: science and politics in early Victorian Britain', Isis (1979), 70, 493-518. Studies on what could be termed the 'travelling sciences' are to be found in S. Cannon: Science in Culture. The Early Victorian Period, New York, 1978, ch. 3. There Cannon introduces the term 'Humboldtian science'. As to the connection between Humboldt and nineteenth-century scientific culture, see M. Dettelbach, 'Humboldtian science', in Cultures of Natural History (ed. N. Jardine, J. A. Secord and E. C. Spary), Cambridge, 1995.

25 Latour, op. cit. (20), 6. 
on heat flows between small bodies to a computation of how much heat the earth receives from the sun. ${ }^{26}$ On the basis of this kind of assumption, elusive phenomena in large dimensions - such as sunshine - can be sufficiently represented in laboratory-sized set-ups and hand-sized inscriptions. ${ }^{27}$

The success bound to the actinometer as a scientific measurement device figures in its primary function: it yielded numerical data; it served in the isolation of a phenomenon; it acted as a focus of experimental inquiry; and it set up a reproducible, multipliable laboratory environment. ${ }^{28}$ The success bound to the experimenter has been one of the main organizing themes of the present analysis. The reading of Herschel's notebooks on the basis of a performance indicates one essential component of scientific research: scientific transcendence from the particular to the general, from the local to the global, is a method based on ruled reasoning and ruled action. Scientific research that successfully conceptualizes and quantifies an elusive phenomenon such as sunshine demands components of two different kinds: it demands measurement equipment, mathematical deduction, and a theoretical idea of how a natural phenomenon interacts with the equipment; but it also demands a very local, labour-intensive and sophisticated process of making the natural phenomenon interact with the experimenter and the artefact in the first place.

\section{Instructions for officers of Her Majesty's Navy}

The most delicate demand imposed on the performance-and-interpretation historiography I have developed so far appears to be the analysis of published instructions for the use of the actinometer - the analysis of a document that displays measurement exclusively as a sequence of determined, replicable manipulations that lead to meaningful measurement. This text not only clashes with my 'practical understanding' but it also reconceals the elements of experimental practice elaborated in the preceding analysis of Herschel's notebooks: it reconceals both the procedures that precede the state of controlled manipulation and the temporality of such a state.

Invoking Wilson's third hermeneutic stance again suggests a starting point for a historical interpretation of this document. How did the printed instructions come into being? How can we historically explain their existence? There is, obviously, the experimental activity itself as a reason for the instruction's existence. Yet public directions for the proper handling of an object emerge from a historical process larger than just the

26 J. H. Lambert, Pyrometrie, Berlin, 1776, quoted in D. S. L. Cardwell, From Watt to Clausius. The Rise of Thermodynamics in the Early Industrial Age, Ithaca and London, 1971, 90.

27 One further example of success in linking together different settings of heat phenomena with the means of mathematical abstraction is Fourier's Analytical Theory of Heat, which was first published in 1822. Karin Knorr coins the terms 'reconfiguration', 'enhancement of nature' and 'enhancement of human agents' to describe how transformations of natural phenomena account for success in science. See K. Knorr Cetina, 'The couch, the cathedral and the laboratory: on the relationship between experiment and laboratory in science', in Pickering, op. cit. (2), 113-38.

28 Taking his efforts in organizing international astronomical observatories as a model, Herschel indeed aimed at setting up a world-wide network of meteorological measurement. See S. Schaffer, 'Astronomers mark time', Science in Context (1988), 2, 115-45; Herschel, op. cit. (8); and his co-operation with James Forbes, Forbes Correspondence, University of St Andrews, nos. 1836/60, 1840/34, 1840/38. 
practice with that object. Published instructions mean manifold replication of a set of actions that was, formerly, unique to John Herschel. The transition from representation of actinometric measurement in personal notebooks - where private, protected labour with an object gradually turns into goal-directed manipulation of this object as a measurement device - to representation in a manual for scientific inquiry is the transition not only from private to public spaces but a shift in the instrument's function and in the character of the labour being performed with it.

In my own performance, I encountered this transition from 'chaotic' to 'directed' labour in a variety of ways. The complementary qualities of scientific practice figured as failure and loss of meaning on the one hand, and as a sequence of meaningful actions on the other. What distinguishes my lab report from a sequence of meaningful actions as described in instructions? I acquired meaningful measurement through perceiving elements that do not belong to the sequence character of goal-directed action, and I deleted these elements rather than following instructions and thus naturally arriving at meaningful measurements. The determinable sequence was not the result of following rules but the result of ignoring and mastering what is not successive and determinable.

The link between the ordered and disordered qualities of scientific practice, and the link between representations in a notebook and in published instructions, perhaps lie in the performance: the labour and the human agency are a vehicle to transform a set of practices into a sequence of instructions. A sequence of instructions is a set of practices in a distinctive state of order and significance: experimental practice as such does not seem to be inherently meaningful. Yet labour and agency as causes of order and meaning eventually disappear from a written sequence of meaningful action.

Interpreting the representation of practice as a linear sequence of meaningful action against the background of performance and within the third hermeneutic stance results in a double-layered answer to the question 'How do instructions come into being?' An instructive text describes meaningful, determined actions. The actions are, therefore, a cause of the emergence of this text. Meaningful actions, however, are themselves a product of a further historical process: they are generated by the experimenter from a state of absence of meaning, and they are temporal both in their emergence and in their existence. A practical interpretation of practice avoids the assumption of inherent regularity and order in experimental action and reads public instructions as a distinguished representation of practice, as the result of labour and as the necessity of displaying practice as orderly, once it is made public. The publication of instructions for an instrument's use implies two items to be historically explained, the interest in disseminating practices and the necessity of creating and maintaining order.

In conclusion, my main argument runs as follows. The foundations of my interpretive practices lie in a two-step interpretive procedure. As a first step, I interpret 'past doing' by means of 'present doing'. In the following step, I interpret 'past doing' by means of present-day 'reading', whereby the present doing feeds into this reading. In this paper, I first displayed the intricacies of 'present doing'. The main result of my practical work with the actinometer turned out to be an underlying contradiction between my labour and its goal: I was performing chaotic operations, and I was supposed to arrive at meaningful, successive measurement. I then interpreted written representations of experimental 
practice along the lines of two exemplary kinds of text: sections of Herschel's personal notebooks and published instructions for the actinometer's use. The result of this interpretation was three different shifts in historical analysis of experimental practice: a shift in the idea of the relationship between the procedure of scientific practice and its written result; a shift in the assumption of order and meaning in successive series of human action; and a shift of the explanatory burden for successful quantification of the sun. I have tried to argue how and to what extent scientific practice is a fundamental epistemic device in the laboratory. In the light of a performed historical study, successful stabilization of experimental settings as a historical item to be explained appears as the product of human action. 